\title{
Partial classification of modules for Lie-algebra of diffeomorphisms of $d$-dimensional torus
}

\author{
S. Eswara Rao \\ School of Mathematics \\ Tata Institute of Fundamental Research \\ Homi Bhabha Road \\ Mumbai 400005 \\ India \\ email: senapati@math.tifr.res.in
}

\begin{abstract}
We consider the Lie-algebra of the group of diffeomorphisms of a $d$ dimensional torus which is also known to be the algebra of derivations on a Laurent polynomial ring $A$ in $d$ commuting variables denoted by $\operatorname{Der} A$. The universal central extension of Der $A$ for $d=1$ is the so called Virasoro algebra. The connection between Virasoro algebra and physics is well known. See for example the book on Conformal Field Theory by Di Francesco, Mathieu and Senechal.

In this paper we classify $(A$, Der $A$ ) modules which are irreducible and has finite dimensional weight spaces. Earlier Larsson constructed a large class of modules the so called tensor fields based on $g \ell_{d}$ modules which are also $A$ modules. We prove that they exhaust all $(A, \operatorname{Der} A)$ irreducible modules.
\end{abstract}




\section{Introduction}

It is well known that the group of diffeomorphisms on a manifold is very important and shows up directly in many branches of physics (see for example Ref.22). We are particularly interested in $d$-dimensional torus. The case $d=1$ is well studied by both mathematicians and physicists. The one dimensional central extension of the Lie-algebra of diffeomorphisms of the circle is well known object called Virasoro algebra. The representation theory of Virasoro algebra is studied in great detail. See Ref.13.

The Virasoro algebra acts on any (except when the level is negative of dual Coxeter number) highest weight module of the affine Lie algebra through the use of the famous Sugawara operators. It is well known that affine Lie algebras admit representation on the Fock space (see Ref.12) and hence admits a representation of the Virasoro algebra. This classical theory is what we originally want to generalize to $d$-dimensional torus.

The relation to physics is well established in the book on Conformal Field Theory by Di Francesco, Mathieu and Senechal. Ref.10. Specially the chapters 13 to 18 explains the connection between physics and the representation theory of Virasoro and affine Kac-Moody Lie-algebras. Several important papers on these aspects have been put in one volume by Goddard and Olive in Ref.8. The most fundamental paper in this direction is due to Belavin, Polyakov and Zamolodchikov in Ref.1.

The generalization of affine Lie algebra is the so called toroidal Lie-

algebra. For the first time a large class of representations are constructed in Refs. 6 and 20 through the use of vertex operators generalizing the Fock space construction to the toroidal Lie-algebras. One significant difference for the toroidal Lie-algebra is that the universal center is infinite dimensional unlike in the affine case where it is one dimensional. 
So the next natural question is to generalise Virasoro algebra and see whether the algebra acts on the Fock space. For that we first denote the Lie algebra of diffeomorphisms of $d$-dimensional torus by DerA (it is known that DerA is isomorphic to the derivations of Laurent polynomial ring A in $\mathrm{d}$-variables). Here one should mention that several attempts has been made by physicists to give a Fock space representation to DerA or to its extension (see Ref. 9). They all failed to produce any interesting results due to lack of proper definition of "normal ordering" among other things. At this juncture an interesting result has come out from Ref.21 which says that DerA has no non-trivial central extension for $d \geq 2$.

Let us go back to the vertex construction of toroidal Lie-algebra of Ref. 6. Here operators are constructed for DerA generalizing the Sugawara Construction. But the corresponding extension for DerA is very wild (certainly non-central) and not tractable (see Ref.5). In the process an interesting abelian extension for DerA has been created in Ref. 6 and the abelian part is exactly the center of the toroidal Lie algebra. So the semi-direct sum of the toroidal Lie algebra and DerA with common extension has emerged as an interesting object which we will now define.

We will first define toroidal Lie algebra. Let $\mathcal{G}$ be simple finite dimensional Lie algebra and let $<,>$ be a non-degenerate symmetric bilinear form on $\mathcal{G}$. Fix a positive integer $\mathrm{d}$ and let $A=\mathbb{C}\left[t_{1}^{ \pm 1}, \cdots, t_{d}^{ \pm 1}\right]$ be a Laurent polynomial ring in $\mathrm{d}$-commuting variables.

Let $\Omega_{A}$ be the module of differentials which can be defined as vector space spanned by $t^{r} K_{i}, i=1, \cdots, d$ and $r \in \mathbb{Z}^{n}$. Let $d_{A}$ be the subspace spanned by $\Sigma r_{i} t^{r} K_{i}$ and consider the toroidal Lie algebra $\mathcal{G} \otimes A \oplus \Omega_{A} / d_{A}$ with Lie-bracket.

$$
\left[X \otimes t^{r}, Y \otimes t^{s}\right]=[X, Y] \otimes t^{r+s}+<X, Y>\Sigma r_{i} t^{r+s} K_{i}
$$

$\Omega_{A} / d_{A}$ is central. 
Let DerA be the derivation on A. For $u \in \mathbb{C}^{d}$ and $r \in \mathbb{Z}^{d}$ let $D(u, r)=$ $\Sigma u_{i} t^{r} t_{i} \frac{d}{d t i}$ where $u=\left(u_{1}, \cdots, u_{d}\right)$ and $r=\left(r_{1}, \cdots, r_{d}\right), t^{r}=t_{1}^{r_{1}} t_{2}^{r_{2}} \cdots t_{d}^{r_{d}} \in A$. Let $K(u, r)=\Sigma u_{i} t^{r} K_{i}$. Consider the Lie-algebra

$$
\begin{gathered}
\tau=\mathcal{G} \otimes A \oplus \Omega_{A} / d_{A} \oplus \operatorname{Der} A \\
{[D(u, r), D(v, s)]=D(w, r+s)-(u, s)(v, r) K(r, r+s)}
\end{gathered}
$$

where $w=(u, s) v-(v, r) u$ and $($,$) is the standard inner product in \mathbb{C}^{d}$

$$
\begin{gathered}
{[D(u, r), K(v, s)]=(u, s) K(v, r+s)+(u, v) K(r, r+s)} \\
{[K(u, r), K(v, s)]=0}
\end{gathered}
$$

The first question is that can we construct a representation for $\tau$ from known methods. Several attempts have been made (Ref. 2, 3 and 6). Eventually in a remarkable paper Yuly Billig (Ref.24) has succeeded in constructing a class of modules for $\tau$ through the use of Vertex operator algebras (VOA). In the process Yuly Billig has used the DerA modules constructed in Ref.16 and studied in Ref.4. One natural question is, does there exists more modules for DerA so that we get a much larger class of models of $\tau$.

In an interesting paper by Jiang and Meng (Ref.11) it is proved that classification of irreducible integrable modules of $\tau$ can be reduced to the classification of irreducible (A, DerA) modules which the current paper settles. See also ref.7 for more precise results.

Let me explain the results of this paper in more detail. In Ref.16, Larsson constructed a large class of DerA modules and some of them with finite dimensional weight spaces. In fact he constructed a functor from $g l_{d}$-modules to DerA-modules. In Ref.4 the author proves that the image of an irreducible finite dimensional module is most often irreducible.

Further Larsson's DerA modules are A modules too and they are always irreducible as (A, DerA)-modules. Thus the purpose of the paper is to prove 
the converse. So we prove in Theorem 1.9 that any (A, DerA) module which is irreducible and has finite dimensional weight spaces has to come from Larsson's construction.

It will be certainly interesting to classify all irreducible DerA modules with finite dimensional weight spaces. Now some kind of highest weight modules are constructed in Ref.2 and 3 (They are not A modules). We will also note that $G L(d, \mathbb{Z})$ acts as automorphisms on DerA and so we can twist a DerA module by $G L(d, \mathbb{Z})$. (Larsson's modules are closed under $G L(d, \mathbb{Z})$ action). So it will be interesting to prove that any irreducible DerA module with finite dimensional weight spaces is either an highest weight module or a Larsson's module upto a twist of $G L(d, \mathbb{Z})$ action. The problem is completely solved for $\mathrm{d}=1$ by Mathieu in Ref.19

Our results in Ref.6 have been reinterpreted in the language of physics by Larsson in Refs. 17 and 18. Larsson is also first to talk about non-central extensions is Ref. 15. DerA has also been studied in Ref.23.

\section{Section}

(1.1) Throughout this paper we fix a positive integer $d \geq 2$ and a Laurent polynomial ring $A=\mathbb{C}\left[t_{1}^{ \pm 1}, \cdots t_{d}^{ \pm 1}\right]$ in $d$ commuting variables. Let $\mathbb{C}^{d}$ be $d$ copies of complex field $\mathbb{C}$. Let $e_{1}, \cdots e_{d}$ be the standard basis of $\mathbb{C}^{d}$ and let $($,$) be the standard form on \mathbb{C}^{d}$ such that $\left(e_{i}, e_{j}\right)=\delta_{i j}$.

(1.2) Let $\Gamma=\mathbb{Z} e_{1} \oplus \cdots \oplus \mathbb{Z} e_{d}$. Throughout this paper we use $m, n, r$ and $s$ to denote elements of $\Gamma$. For $r=\sum r_{i} e_{i} \in \Gamma$ let $t^{r}=t_{1}^{r_{1}} t_{2}^{r_{2}} \cdots t_{d}^{r_{d}} \in A$ and let $D^{i}(r)=t^{r} t_{i} \frac{d}{d t_{i}}$ be a derivation on $A$. Let Der $A$ be the Lie-algebra of derivations of $A$. It is easy to verify that $D^{i}(r), 1 \leq i \leq d, r \in \Gamma$ is a basis of Der $A$. For $u=\sum u_{i} e_{i} \in \mathbb{C}^{d}$ let $D(u, r)=\sum u_{i} D^{i}(r)$. Then $\operatorname{Der} A$ has the following Lie structure: 
$(1.3)$

$$
[D(u, r), D(v, s)]=D(w, r+s)
$$

where $w=(u, s) v-(v, r) u, r, s \in \Gamma$ and $u, v \in \mathbb{C}^{d}$. Let $h$ be the subspace spanned by $D^{i}(0), 1 \leq i \leq d$, which is a maximal abelian subalgebra of $\operatorname{Der} A$.

(1.4) Note that $D(u, r) t^{s}=(u, s) t^{r+s}$. Thus $A \oplus \operatorname{Der} A$ is a Lie-algebra by extending the Lie structure in the following way

$$
\begin{gathered}
{\left[t^{r}, t^{s}\right]=0} \\
{\left[D(u, r), t^{m}\right]=(u, m) t^{r+m} .}
\end{gathered}
$$

Let $\tilde{h}=\mathbb{C} \oplus h$ which is an abelian subalgebra of $A \oplus \operatorname{Der} A$.

The purpose of this paper is to study $A \oplus \operatorname{Der} A$ modules which are weight modules for $\tilde{h}$ with finite dimensional weight spaces and to classify such modules with some natural conditions.

We first recall Der $A$ modules which are constructed and studied in References 16 and 4 .

(1.5) Let $g \ell_{d}$ be the Lie-algebra of $d \times d$ matrices with entries in $\mathbb{C}$. Let $E_{i j}$ be the elementary matrix with $(i, j)$ th entry 1 and zero elsewhere. Then it is well known that $g \ell_{d}$ is spanned by $E_{i j}, 1 \leq i, j \leq d$ with the following Lie-bracket

$$
\left[E_{i j}, E_{k \ell}\right]=\delta_{j k} e_{i \ell}-\delta_{i \ell} E_{k j} .
$$

Let $g \ell_{d}=s \ell_{d} \oplus \mathbb{C} I$ where $s \ell_{d}$ is a Lie-subalgebra of trace zero matrices and $I$ is the identity matrix. Let $V(\psi)$ be the irreducible finite dimensional module for $s \ell_{d}$ where $\psi$ is a dominant integral weight. Let $I$ act by scalar $b$ on $V(\psi)$ and denote the resultant $g \ell_{d}$ module by $V(\psi, b)$. Let $\alpha \in \mathbb{C}^{d}$ and we will make $F^{\alpha}(\psi, b):=V(\psi, b) \otimes A$ a $\operatorname{Der} A$ module. First denote $v \otimes t^{m}$ by $v(m)$ for $v$ in $V(\psi, b)$ and $m$ in $\Gamma$. 
(1.6) Definition (Ref. 16)

$$
D(u, r) \cdot v(m)=(u, m+\alpha) v(m+r)+\left(\sum_{i, j} u_{i} r_{j} E_{j i} v\right)(m+r)
$$

where $m, r \in \Gamma, u \in \mathbb{C}^{d}, v \in V(\psi, b)$. We will now recall the following

(1.7) Theorem (Theorem (1.9) and Proposition (5.1) of Ref.4).

(1) $F^{\alpha}(\psi, b)$ is irreducible as $\operatorname{Der} A$ module if $(\psi, b) \neq\left(\delta_{k}, k\right),(0, b), 1 \leq k \leq$ $d-1$ where $\delta_{k}$ is the $k$ th fundamental weight of $s \ell_{d}$.

(2) $F^{\alpha}(0, b)$ is irreducible as $\operatorname{Der} A$ module unless $\alpha \in \Gamma$ and $b \in\{0, d\}$.

In all other cases $F^{\alpha}(\psi, b)$ is reducible and the submodule structure has been worked out in Proposition (5.1) and Theorem (5.5) of Ref.4.

Recall that $A$ is associative algebra with unit and $F^{\alpha}(\psi, b)$ is a $A$ module by defining

$$
t^{m} \cdot v(r)=v(m+r)
$$

for $m, r \in \Gamma$ and $v \in V(\psi, b)$. Further it is easy to see that $F^{\alpha}(\psi, b)$ is a $A \oplus \operatorname{Der} A$ module.

(1.8) Proposition $F^{\alpha}(\psi, b)$ is irreducible as $A \oplus \operatorname{Der} A$ module.

Proof First note that $F^{\alpha}(\psi, b)$ is a weight module with respective to $\tilde{h}$ and the weight spaces are $V(\psi) \otimes t^{m}$. Suppose $W$ is a non-zero $A \oplus \operatorname{Der} A$ submodule of $F^{\alpha}(\psi, b)$. As submodule of a weight module is a weight module, $W$ is a weight module. From the action of $A$ it is clear that $v(m) \in W$ implies $v(s) \in W$ for all $s \in \Gamma$. Thus $W=W_{1} \otimes A$ for some $W_{1} \subseteq V(\psi, b)$. Now choose $u=e_{i}, r=e_{j}$ and consider

$$
D(u, r) v(m)=(u, m+\alpha) v(m+r)+\left(E_{j i} v\right)(m+r) .
$$

It now follows from the above remarks that $W_{1}$ is $g \ell_{d}$-invariant. Since $V(\psi)$ is irreducible and $W_{1}$ is non-zero, it follows that $W_{1}=V(\psi)$ and hence $W=F^{\alpha}(\psi, b)$. 
The purpose of this paper is to prove converse of the above proposition. In other words we classify $A \oplus \operatorname{Der} A$ modules with certain natural properties.

(1.9) Theorem Let $V$ be irreducible module for $A \oplus \operatorname{Der} A$ which is also a weight module for $\tilde{h}$ with finite dimensional weight spaces. We further assume the following:

(1) $V$ is a $A$-module as associative algebra and the Lie-module structure of $A$ comes from associative algebra.

(2) $1 . v=v, \forall v$ in $V$.

Then $V \cong F^{\alpha}(\psi, b)$ (for some $\left.\alpha, \psi, b\right)$ as $A \oplus \operatorname{Der} A$-module.

We need to develop several lemmas to prove the theorem which will be done in Section 2. The final proof will be given in Section 3.

\section{Section}

First we need to change some notation. We treat $A$ as group algebra over $\Gamma$. For that let $k(r)$ be a symbol for $r \in \Gamma$. Let $A$ be the linear span of $k(r), r \in \Gamma$ with multiplication defined as $k(r) \cdot k(s)=k(r+s)$.

Let $U$ be the universal enveloping algebra of $A \oplus \operatorname{Der} A$. Let $L$ be the two sided ideal of $U$ generated by $k(r) k(s)-k(r+s)$ and $k(0)-1$.

Throughout this section the module $V$ is as in Theorem (1.9). Since $V$ is a $A$ module, $L$ acts trivially and hence $V$ is a $U / L$-module. Let $V=\oplus_{r \in \Gamma} V_{r}$ be the weight space decomposition and $V_{r}=\{v \in V \mid D(u, 0) v=(u, r+\alpha) v, \forall u$ in $\left.\mathbb{C}^{d}\right\}$. Such a uniform $\alpha$ in $\mathbb{C}^{d}$ exists as $V$ is irreducible. In fact take any weight space where $h$ acts as linear function which can be taken as $u \mapsto(u, \alpha)$ for some $\alpha \in \mathbb{C}^{d}$. Because of irreducibility the action of $h$ on the rest of the 
spaces is easily computed. Further each $V_{r}$ is a $\tilde{h}$-module as 1 in $A$ acts as one on the entire module.

(2.1) Let $U_{1}=U / L$ and let $T(u, r)=k(-r) D(u, r)-D(u, 0)$ as an element of $U_{1}$ for $u \in \mathbb{C}^{d}$ and $r \in \Gamma$. Let $T$ be the subspace spanned by $T(u, r)$ for all $u$ and $r$.

\section{(2.2) Proposition}

(1) $[T(v, s), T(u, r)]=(u, s) T(v, s)-(v, r) T(u, r)+T(w, r+s)$ where $w=(v, r) u-(u, s) v$ and hence $T$ is a Lie-subalgebra.

(2) $[D(v, 0), T(u, r)]=0$

(3) $V=\oplus V_{r}$ be weight space decomposition. Then each $V_{r}$ is $T$-invariant

(4) Each $V_{r}$ is $T$-irreducible

(5) $V_{r} \cong V_{s}$ as $T$-module.

\section{Proof}

(2) $[D(v, 0), k(-r) D(u, r)-D(u, 0)]$

$$
\begin{aligned}
& =[D(v, 0), k(-r) D(u, r)] \\
& =[D(v, 0), k(-r)] D(u, r) \\
& +k(-r)[D(v, 0), D(u, r)] \\
& =-(v, r) k(-r) D(u, r) \\
& +(v, r) k(-r) D(u, r) \\
& =0 .
\end{aligned}
$$

(1) From (2) it follows that

$$
[T(v, s), T(u, r)]=[k(-s) D(v, s), k(-r) D(u, r)]
$$




$$
\begin{aligned}
=[k(-s), & k(-r) D(u, r)] D(v, s)+k(-s)[D(v, s), k(-r) D(u, r)] \\
= & {[k(-s), k(-r)] D(u, r) D(v, s) } \\
+ & k(-r)[k(-s), D(u, r)] D(v, s) \\
+ & k(-s)[D(v, s), k(-r)] D(u, r) \\
+ & k(-s) k(-r)[D(v, s), D(u, r)] \\
= & (u, s) k(-s) D(v, s) \\
& -(v, r) k(-r) D(u, r) \\
+ & k(-s-r) D(w, r+s) \text { where } w=(v, r) u-(u, s) v \\
= & (u, s) T(v, s) \\
& -(v, r) T(u, r) \\
+ & T(w, r+s) \\
= & (v, r) u-(u, s) v
\end{aligned}
$$

(3) From (2) it follows that $T$ commutes with $h$ and hence $V_{r}$ is a $T$-module.

(4) Let $U=\oplus_{r \in \Gamma} U_{r}$ where $U_{r}=\left\{v \in U \mid[D(u, 0), v]=(u, r) v\right.$ for $\left.u \in \mathbb{C}^{d}\right\}$. Since $V$ is $A \oplus \operatorname{Der} A$ irreducible for $v, w$ in $V_{r}$ there exists $X$ in $U_{0}$ such that $X v=w$. This is due to weight reasons. Now $X=\sum a_{i} X_{i}$ where each $X_{i}$ is of the form $k(-r) D\left(u_{0}, r_{1}\right) \cdots D\left(u_{k}, r_{k}\right)$ where $\sum r_{i}=r$. We are using the fact that $L$ acts trivially on $V$. Now using the fact that $k(-s) D(u, r)=$ $D(u, r) k(s)-(u, s) D(u, r)$ and the fact that $k(r) k(s)=k(r+s)$ we see that each $X_{i}$ is linear combination of elements of the form

$$
k\left(-r_{1}\right) D\left(u_{1}, r_{1}\right) k\left(-r_{2}\right) D\left(u_{2}, r_{2}\right) \cdots k\left(-r_{k}\right) D\left(u_{k}, r_{k}\right)
$$

This proves $X \in U(T)$, the universal enveloping algebra of $T$. Hence $V_{r}$ is $T$ irreducible.

(5) First note that $k(s-r) V_{r} \subseteq V_{s}$. Repeating the same we see that

$$
V_{r}=k(r-s) k(s-r) V_{r} \subseteq k(r-s) V_{s} \subseteq V_{r}
$$


Thus $V_{r}=k(r-s) V_{s}$. Define $f: V_{r} \rightarrow V_{s}$ by $f(v)=k(s-r) v$ which is clearly injective and surjective. Now

$$
\begin{aligned}
f(T(u, k) v) & =k(s-r) T(u, k) v \\
& =T(u, k) k(s-r) v \\
& =T(u, k) f(v)
\end{aligned}
$$

Thus $f$ is a $T$-homomorphism. This proves (5).

(2.3) Notation For any integer $k>0, r, m_{1}, \cdots m_{k} \in \Gamma$ define

$$
\begin{aligned}
& T_{k}\left(u, r, m_{1}, \cdots m_{k}\right)=T(u, r)-\sum_{i} T\left(u, r+m_{i}\right)+\sum_{i<j} T\left(u, r+m_{i}+m_{j}\right) \cdots \\
& (-1)^{j} \sum_{1 \leq i_{1}<i_{2}<\cdots<i_{j} \leq k} T\left(u, r+m_{i_{1}}+\cdots+m_{i_{j}}\right) \cdots(-1)^{k} T\left(u, r+m_{1}+m_{2}+\cdots+m_{k}\right) .
\end{aligned}
$$

Let $I_{k}$ be the linear span of $T_{k}\left(u, r, m_{1} \cdots m_{k}\right), u \in \mathbb{C}^{d}$ for all $r, m_{1}, m_{2} \cdots m_{k} \in$ $\Gamma$.

(2.4) Lemma (1) $T_{k}\left(u, r, m_{1}, \cdots m_{k}\right)=T_{k}\left(u, r, m_{\sigma(1)}, \cdots m_{\sigma(k)}\right)$ for any permutation $\sigma$ on $k$-letters.

(2) $T_{k}\left(u, r, m_{1}, \cdots m_{k}\right)=T_{k-1}\left(u, r, m_{1}, \cdots m_{k-1}\right)-T_{k-1}\left(u, r+m_{k}, m_{1}, \cdots m_{k-1}\right)$

(3) $I_{k}$ is an ideal of $T$.

(4) $I_{k} \subseteq I_{k-1}$ for $k \geq 2$.

(5) $\left[I_{k}, I_{\ell}\right] \subseteq I_{k+\ell-1}$ for $k, \ell \geq 1$.

Proof (1) Follows from definition

(2) Collect all terms where $m_{k}$ does not occur in the sum of $T_{k}$ and that can be seen to be equal to $T_{k-1}\left(u, r, m_{1}, \cdots m_{k-1}\right)$. Sum of the rest of the terms can be seen to equal to $-T_{k-1}\left(u, r+m_{k}, m_{1}, \cdots m_{k-1}\right)$. This is because every term contains $m_{k}$ 
(3) $\left[T(v, s), T_{k}\left(u, r, m_{1}, \cdots m_{k}\right)\right]=$

$$
\begin{gathered}
(u, s) \sum_{\ell=0}^{k}\left(\begin{array}{c}
k \\
\ell
\end{array}\right)(-1)^{\ell} k(-s) D(v, s) \\
-(v, r) T_{k}\left(u, r, m_{1}, \cdots m_{k}\right) \\
+\sum\left(v, m_{i}\right) T_{k-1}\left(u, r+m_{i}, m_{1}, \cdots \widehat{m_{i}}, \cdots m_{k}\right) . \\
+(v, r) T_{k}\left(u, r+s, m_{1}, \cdots m_{k}\right) \\
-\sum\left(v, m_{i}\right) T_{k-1}\left(u, r+s+m_{i}, m_{1}, \cdots \widehat{m_{i}}, \cdots m_{k}\right) \\
-(u, s) T_{k}\left(v, r+s, m_{1}, \cdots m_{k}\right) .
\end{gathered}
$$

By applying Proposition $2.2(1)$ write $\left[T(v, s), T_{k}\left(u, r, m_{1}, \cdots m_{k}\right)\right]=A_{1}+$ $A_{2}+A_{3}$. It is easy to see that $A_{1}$ is the first term of the above formula. Now in $A_{2}$ look for the terms where $\left(v, m_{i}\right)$ occurs and that can be seen as a component of the third term of the above formula. Now in $A_{2}$ the terms where no $m_{i}$ occurs is equal to the second term of the above formula. The rest of the formula can be seen in a similar way. This proves the claim. Now note that the first term in the claim is zero. Clearly 2nd, 4th and 6th terms are in $I_{k}$. Now 3rd and 5 th term is equal to

$$
\begin{aligned}
& \sum\left(v, m_{i}\right) T_{k-1}\left(u, r+m_{i}, m_{1}, \cdots \widehat{m_{i}}, \cdots m_{k}\right) \\
- & \sum\left(v, m_{i}\right) T_{k-1}\left(u, r+m_{i}+s, m_{1} \cdots \widehat{m_{i}}, \cdots m_{k}\right) \\
= & \sum\left(v, m_{i}\right) T_{k}\left(u, r+m_{i}, m_{1}, \cdots \widehat{m_{i}} \cdots m_{k}, s\right)
\end{aligned}
$$

(by Lemma $2.4(2)$ ).

(4) Follows from (2).

$$
\begin{aligned}
& {\left[T_{\ell}\left(v, s, n_{1}, \cdots n_{\ell}\right), T_{k}\left(u, r, m_{1}, \cdots m_{k}\right)\right]} \\
& \sum_{t=0}^{\ell} \sum_{i_{1}<i_{2}<\cdots<i_{t}}\left(u, s+n_{i_{1}}+\cdots+n_{i_{t}}\right) .
\end{aligned}
$$




$$
\begin{gathered}
\sum_{b=0}^{k}\left(\begin{array}{l}
k \\
b
\end{array}\right)(-1)^{b} T\left(v, s+n_{i_{1}}+\cdots n_{i_{t}}\right) \\
-\sum_{t=0}^{k} \sum_{j_{1}<\cdots<j_{t}}\left(v, r+m_{j_{1}}+\cdots+m_{j_{t}}\right) . \\
\sum_{b=0}^{\ell}\left(\begin{array}{l}
\ell \\
b
\end{array}\right)(-1)^{b} T\left(u, r+m_{i_{1}}+\cdots+m_{i_{t}}\right) \\
+(v, r) T_{k+\ell}\left(u, r+s, m_{1}, \cdots m_{k}, n_{1}, \cdots n_{\ell}\right) \\
-(u, s) T_{k+\ell}\left(v, r+s, m_{1}, \cdots m_{k}, n_{1}, \cdots n_{\ell}\right) \\
-\sum\left(v, m_{i}\right) T_{k+\ell-1}\left(u, r+s+m_{i}, m_{1}, \cdots \widehat{m_{i}}, \cdots m_{k}, n_{1}, \cdots n_{\ell}\right) \\
+\sum\left(u, n_{j}\right) T_{k+\ell-1}\left(v, r+s+n_{j}, m_{1}, \cdots m_{k}, n_{1}, \cdots \widehat{n}_{j}, \cdots n_{\ell}\right)
\end{gathered}
$$

The above formula can be deduced as in (3) from Proposition 2.2 (1). Now note that the first two terms are zero as

$$
\sum_{b=0}^{k}\left(\begin{array}{l}
k \\
b
\end{array}\right)(-1)^{b}=0=\sum_{b=0}^{\ell}\left(\begin{array}{l}
\ell \\
b
\end{array}\right)(-1)^{b} .
$$

The rest of the four terms are in $I_{k+\ell-1}$ and this proves (5).

(2.5) Lemma For $u \in \mathbb{C}^{d}, 0 \neq m_{i} \in \Gamma, s \in \Gamma$.

(1) $T_{k}\left(u, s, m_{1}, \cdots m_{k}\right) \notin I_{k+1}$ for $k \geq 1$.

(2) $T_{k}\left(u, s, m_{1}, \cdots m_{k}\right)$

$$
+T_{k}\left(u, s, n, m_{2}, \cdots m_{k}\right)=T_{k}\left(u, s, m_{1}+n, m_{2}, \cdots m_{k}\right)+I_{k+1}
$$

(3) $T_{k}\left(u, s,-m_{1}, m_{2}, \cdots m_{k}\right)$

$$
=-T_{k}\left(u, s-m_{1}, m_{1}, m_{2}, \cdots m_{k}\right)
$$


Proof To prove the Lemma, we first interpret $T_{k}$ 's as certain polynomials in $A=\mathbb{C}\left[t_{1}^{ \pm 1}, \cdots t_{d}^{ \pm 1}\right]$. We fix a non-zero $u$ in $\mathbb{C}^{d}$. Let $k$ be a positive integer and let $m_{1}, m_{2}, \cdots m_{k} \in \Gamma$. Let $P_{k}\left(m_{1}, \cdots m_{k}\right)=\prod_{1 \leq i \leq k}\left(1-t^{m_{i}}\right)$. Recall $t^{m_{i}}=t_{1}^{\left(m_{i}\right)_{1}} \cdots t_{d}^{\left(m_{i}\right)_{d}}$. Let $J_{k}$ be the ideal in $A$ generated by $P_{k}\left(m_{1}, \cdots m_{k}\right)$ for all non-zero $m_{i}$ 's $\in \Gamma$. Then clearly $J_{k+1} \subseteq J_{k}$. It is easy to see that $T_{k}\left(u, r, m_{1}, \cdots m_{k}\right)$ can be identified with polynomial $t^{r} P_{k}\left(m_{1}, \cdots m_{k}\right)$. Recall that $u$ is fixed.

Thus it is sufficient to prove that,

Claim $1 \quad P_{k}\left(m_{1}, \cdots m_{k}\right) \notin J_{k+1}$. Suppose

$$
P_{k}\left(m_{1}, \cdots m_{k}\right)=\sum f_{\ell} P_{k+1}\left(n_{\ell_{1}}, n_{\ell_{2}}, \cdots n_{\ell_{k+1}}\right)
$$

where $f_{\ell} \in A$. Let $D t_{i}=t_{i} \frac{d}{d t_{k}}$. Now consider $D_{t_{i_{1}}} \cdots D_{t_{i_{k}}} P_{k+1}\left(n_{1}, \cdots n_{k+1}\right)$ and evaluate at $\left(t_{1}, \cdots t_{d}\right)=(1, \cdots 1)$. This can be seen to be zero as after differentiating $P_{k+1}, k$ times, each component has at least one factor $\left(t^{n_{i}}-1\right)$. We will now prove that there exists $i_{1}, \cdots i_{k}$ such that

Claim $\left.2 D_{t_{i_{1}}} \cdots D_{t_{i_{k}}} P_{k}\left(m_{1}, \cdots m_{k}\right)\right|_{t=(1, \ldots 1)}$ is non-zero. Thus $*$ can not hold. This prove the claim 1 . Now choose $\ell, 1 \leq \ell \leq d$ such that $S=\{i \mid$ $\left.\left(m_{i}\right)_{\ell} \neq 0\right\}$ is non-empty. Let $\# S=p$ and let $i_{1}, \cdots i_{p} \in S$. Consider

$$
\left(D_{t_{\ell}}\right)^{p} P_{k}\left(m_{1}, \cdots m_{k}\right)=\mu \prod_{i \in S}\left(m_{i}\right)_{\ell} \prod_{j \notin S}\left(1-t^{m_{j}}\right) t^{m_{i_{1}}+m_{i_{2}}+\cdots+m_{i_{p}}}+J_{k-s+1}
$$

which is not too difficult to see. Where $\mu$ is a non-negative integer. Repeating the process finitely many times (choosing different index $\ell^{1} \neq \ell$ ). We see that there exists $i_{1}, \cdots, i_{k}$ such that $D_{t_{i_{1}}} D_{t_{i_{2}}} \cdots D_{t_{i_{k}}} \cdot P_{k}\left(m_{1}, \cdots, m_{k}\right)=$ $\lambda t^{m_{1}+\cdots+m_{k}}+J_{1}$ where $\lambda$ is non-zero integer. Now evaluating at $t=(1, \cdots 1)$ we see that claim 2 is true.

To see (2) first note that

$$
\left(1-t^{m}\right)\left(1-t^{n}\right)+\left(1-t^{m+n}\right)=\left(1-t^{m}\right)+\left(1-t^{n}\right) .
$$


Thus

$$
\begin{gathered}
t^{s} \prod_{i=2}^{k}\left(1-t^{m_{i}}\right)\left(1-t^{m_{1}}\right)+t^{s} \prod_{i=2}^{k}\left(1-t^{m_{i}}\right)\left(1-t^{n}\right) \\
=t^{s} \prod_{i=2}^{k}\left(1-t^{m_{i}}\right)\left(1-t^{n}\right)\left(1-t^{m_{1}}\right) \\
+t^{s} \prod_{i=2}^{k}\left(1-t^{m_{i}}\right)\left(1-t^{m_{1}+n}\right) .
\end{gathered}
$$

This proves (2).

(3) is easy to check.

\section{(2.6) Lemma}

$$
\operatorname{Dim}\left(I_{k} / I_{k+1}\right) \leq d^{k+1}, k \geq 1
$$

$$
T=I_{1}
$$

In particular $I_{k}$ is a co-finite ideal in $T$.

Proof First note that from Lemma 2.4(2) we have

$$
T_{k}\left(u, r, m_{1}, \cdots m_{k}\right)=T_{k}\left(u, s, m_{1} \cdots m_{k}\right) \bmod I_{k+1}
$$

for all $r, s \in \Gamma$. Further

$$
-T_{k}\left(u, 0, m_{1}, \cdots m_{k}\right)=T_{k}\left(u, 0,-m_{1}, m_{2} \cdots m_{k}\right) \bmod I_{k+1}
$$

which follows from above and Lemma 2.5 (3). Now from additive property of Lemma 2.5(2) it follows that $I_{k} / I_{k+1}$ is spanned by $T_{k}\left(u, 0, e_{i_{1}}, \cdots e_{i_{k}}\right)$ where $e_{1}, \cdots e_{d}$ is the standard basis. Thus (1) follows. (2) follows from definitions. Now it is easy to conclude that $I_{k}$ is a co-finite ideal for each $k$. 


\section{Section}

We will explain the plan of the proof of Theorem (1.9). First we will prove that $T / I_{2} \cong g \ell_{d}(\mathbb{C})$. Then we will prove that if $I_{k}, k \geq 2$ is zero on a finite dimensional irreducible module $V$ of $T$ then $I_{2}$ is zero on $V$. Thus $V$ is a module for $T / I_{2} \cong g \ell_{d}(\mathbb{C})$. Further we prove that any co-finite ideal $J$ of $T$ contains $I_{k}$ for large $k$. Thus any irreducible finite dimensional module $V$ of $T$ is actually a module for $T / I_{2}$. From this it will be easy to conclude Theorem 1.9 which will be explained at the end of the section.

(3.1) Proposition $T / I_{2} \cong g \ell_{d}(\mathbb{C})$.

Proof First recall that $F^{\alpha}(\psi, b)$ is a $A \oplus \operatorname{Der} A$-module and each weight space $V(\psi) \otimes t^{m}$ is a $T$-module. It is easy to verify that $I_{2}$ acts trivially on $V(\psi) \otimes t^{m}$. Now note that $T\left(e_{i}, e_{j}\right) v(m)=E_{j i} v(m) \neq 0$ for some $\psi$. From this we conclude that $T\left(e_{i}, e_{j}\right)$ is non-zero in $T / I_{2}$. Now it is easy to see that $T(u, s)+T(u, r)=T(u, r+s) \bmod I_{2}$ and hence $T\left(e_{i}, e_{j}\right)$ spans $T / I_{2}$. Define $\pi: T / I_{2} \rightarrow g \ell_{d}(\mathbb{C})$.

$$
\pi\left(T\left(e_{i}, e_{j}\right)\right)=E_{j i}
$$

Consider

$$
\begin{aligned}
X= & {\left[T\left(e_{i}, e_{j}\right), T\left(e_{k}, e_{\ell}\right)\right] } \\
= & {\left[k\left(-e_{j}\right) D\left(e_{i}, e_{j}\right), k\left(-e_{\ell}\right) D\left(e_{k}, e_{\ell}\right)\right] } \\
= & -\delta_{i \ell} k\left(-e_{\ell}\right) D\left(e_{k}, e_{\ell}\right) \\
& +\delta_{k j} k\left(-e_{j}\right) D\left(e_{i}, e_{j}\right) \\
- & \delta_{k j} k\left(-e_{\ell}-e_{j}\right) D\left(e_{i}, e_{\ell}+e_{j}\right) \\
& +\delta_{i \ell} k\left(-e_{\ell}-e_{j}\right) D\left(e_{k}, e_{\ell}+e_{j}\right) .
\end{aligned}
$$

Follows from Proposition (2.2). Note that the following is true in $T / I_{2}$.

$$
k\left(-e_{\ell}-e_{j}\right) D\left(e_{s}, e_{\ell}+e_{j}\right)=k\left(-e_{\ell}\right) D\left(e_{s}, e_{\ell}\right)
$$




$$
+k\left(-e_{j}\right) D\left(e_{s}, e_{j}\right)-D\left(e_{s}, 0\right)
$$

for $s=i, k$. Thus $X=-\delta_{k j}\left(k\left(-e_{\ell}\right) D\left(e_{i}, e_{\ell}\right)-D\left(e_{i}, 0\right)\right)$

$$
\begin{gathered}
+\delta_{i \ell}\left(k\left(-e_{j}\right) D\left(e_{k}, e_{j}\right)-D\left(e_{k}, 0\right)\right) \\
=-\delta_{k j} T\left(e_{i}, e_{\ell}\right)+\delta_{i \ell} T\left(e_{k}, e_{j}\right) .
\end{gathered}
$$

Thus $\pi$ defines a surjective homomorphism. As $T(u, 0)$ is zero it follows that $T\left(e_{i}, e_{j}\right)$ span $T / I_{2}$ which proves $\operatorname{dim}\left(T / I_{2}\right) \leq d^{2}$. Thus $\pi$ defines an isomorphism.

(3.2) Lemma (Yuly Billig) Suppose $\mathcal{G}$ is a Lie- algebra over $\mathbb{C}$ and $J$ is an ideal with spanning set $J_{\alpha}, \alpha \in B$. Suppose there exists an element $I$ in $\mathcal{G}$ such that $\left[I, J_{\alpha}\right]=\lambda J_{\alpha}, \lambda \neq 0$ for all $\alpha \in B$. then $J$ acts trivially on any irreducible finite dimensional module $V$ of $\mathcal{G}$.

Proof Since the base field is complex numbers and $V$ is finite dimensional, $I$ has eigen vectors. Let $\lambda_{1}, \cdots, \lambda_{k}$ be all the eigen values of $I$ on $V$. Choose $\lambda_{i}$ such that $\lambda+\lambda_{i}$ is not an eigenvalue. Let $v$ be eigenvector with eigenvalue $\lambda_{i}$ for $I$. Consider $I J_{\alpha} v=J_{\alpha} I v+\left[I, J_{\alpha}\right] v=\left(\lambda_{i}+\lambda\right) J_{\alpha} v$. This proves $J_{\alpha} v=0 \forall \alpha \in B$. Let $W=\left\{w \in V \mid J_{\alpha} w=0 \forall \alpha \in B\right\}$. Since $J$ is an ideal, it is easy to see that $W$ is a $\mathcal{G}$-module. But $W \neq 0$. Since $V$ is irreducible $W=V$ which proves that $J$ acts trivially on $V$.

(3.3) Proposition Suppose $V$ is irreducible finite dimensional module for $T$ such that $I_{k+1}$ acts trivially on $V$. Then $I_{2}$ acts trivially on $V$.

Proof From the proof of Lemma 2.4(3) we have

$$
\begin{gathered}
{\left[T(v, s), T_{k}\left(u, r, m_{1}, \cdots m_{k}\right)=-(v, r) T_{k+1}\left(u, r, m_{1}, \cdots m_{k}, s\right)\right.} \\
-(u, s) T_{k}\left(v, r+s, m_{1}, \cdots m_{k}\right)+\sum\left(v, m_{i}\right) T_{k}\left(u, r+m_{i}, m_{1}, \cdots \widehat{m_{i}}, \cdots m_{k}, s\right) .
\end{gathered}
$$


Let $I=\sum T\left(e_{i}, e_{i}\right)$ and note that $I$ is actually identity element in $T / I_{2} \cong$ $g \ell_{d}(\mathbb{C})$. Thus $I$ is non-zero on $T / I_{k+1}$ for $k \geq 1$.

\section{Claim}

$$
\left[I, T_{k}\left(u, r, m_{1}, \cdots m_{k}\right)=(k-1) T_{k}\left(u, r, m_{1}, \cdots m_{k}\right)\right.
$$

Consider

$$
\begin{gathered}
{\left[\sum_{j} T\left(e_{j}, e_{j}\right), T_{k}\left(u, r, m_{1}, \cdots m_{k}\right)=-\sum u_{j} T_{k}\left(e_{j}, r+e_{j}, m_{1}, \cdots m_{k}\right)\right.} \\
+\sum_{i, j}\left(m_{i}\right)_{j} T_{k}\left(u, r+m_{i}, m_{1}, \cdots \widehat{m_{i}}, \cdots m_{k}, e_{j}\right) .
\end{gathered}
$$

Now we use Lemma 2.5(2) and the following facts.

(1) $T_{k}$ is linear in $u$

(2) $T_{k}\left(u, r+m, m_{1}, \cdots m_{k}\right)=T_{k}\left(u, r, m_{1}, \cdots m_{k}\right) \bmod I_{k+1}$ (by lemma $2.4(2))$.

(3) $I_{k+1}$ is zero on $V$.

From that we conclude that

$$
\left[I, T_{k}\left(u, r, m_{1}, \cdots m_{k}\right)\right]=-T_{k}\left(u, r, m_{1}, \cdots m_{k}\right)+k T_{k}\left(u, r, m_{1}, \cdots m_{k}\right)
$$

which proves the claim. Now we can use Lemma (3.2) for the ideal $I_{k}$. Thus $I_{k}$ is zero on $V$. Repeating this argument we conclude that $I_{2}$ acts trivially on $V$. This argument breaks down for $k=1$ as we cannot apply the Lemma 3.2 .

(3.4) Proposition Any co-finite ideal $J$ of $T$ contains $I_{k}$ for large $k$. 
Proof Claim $J \cap I_{k}$ is co-finite in $T$ for all $k$. For that consider $\varphi: T \mapsto$ $T / J \oplus T / I_{k}$

$$
v \mapsto(v, v) .
$$

Clearly ker $\varphi=J \cap I_{k}$ and $T / J \cap I_{k}$ is a subalgebra of finite dimensional Lie-algebra $T / J \oplus T / I_{k}$. This proves the claim.

Consider

$I_{k} / J \cap I_{k} \stackrel{\varphi_{1}}{\longrightarrow} I_{k-1} / J \cap I_{k} \stackrel{\varphi_{2}}{\longrightarrow} I_{k-1} / J \cap I_{k-1}$ where $\varphi_{1}$ is injective and $\varphi_{2}$ is surjective. Let $\rho_{k}=\varphi_{2} \circ \varphi_{1}$. Thus $\rho_{k}: I_{k} / I_{k} \cap J \rightarrow I_{k-1} / J \cap I_{k-1}$. Clearly $\rho_{k}$ in injective. Let $t_{n}=\operatorname{dim} I_{n} / I_{n} \cap J$ and note that $t_{n+1} \leq t_{n}$. Thus $\left\{t_{n}\right\}_{n \in \mathbb{Z}^{+}}$ is a decreasing sequence of non-negative integers. Therefore $t_{k}=s$ for some $s$ and for large $k>N$.

First we note the following two statements for a fixed $i$.

(1) For $\ell \neq i$

$$
\left[T\left(e_{\ell},-e_{\ell}\right), T_{k}\left(e_{i}, 0, e_{j_{1}}, \cdots e_{j_{k}}\right)\right]=-k_{\ell} T_{k}\left(e_{i}, 0, e_{j_{1}}, \cdots e_{j_{k}}\right)
$$

where $k_{\ell}$ is the number of $e_{\ell}$ that occur in $T_{k}\left(e_{i}, 0, e_{j_{1}}, \cdots e_{j_{k}}\right)$.

(2) Suppose the ideal $J$ contains $\sum a_{m, I} T_{m}\left(e_{i}, 0, e_{j_{1}}, \cdots e_{j_{m}}\right)$ where the number of $e_{i}$ 's that occur in $T_{m}\left(e_{i}, 0, e_{j_{1}}, \cdots e_{j_{m}}\right)$ is same for all $m$ where $I=\left\{j_{1}, \cdots j_{k}\right\}$. Then $J$ contains $T_{m}\left(e_{i}, 0, e_{j_{1}}, \cdots e_{j_{m}}\right)$ for $m \ni a_{m, I} \neq 0$.

(1) Follows from the proof of lemma 2.4(3). (2) follows from (1). We will prove the Proposition assuming $d \geq 3$ to avoid some computations. For a fixed $i$, consider the following set

$$
S=\left\{T_{k}\left(e_{i}, 0, e_{j_{1}}, \cdots e_{j_{k}}\right) \mid j_{\ell} \neq i \text { for all } \ell\right\} .
$$

Now choose $k \ni \# S>s$ and $k>N$. Thus $S$ is linearly dependent mod $I_{k} \cap J$. Thus there exists non-zero scalars $a_{I}\left(I=\left\{j_{1}, \cdots j_{k}\right\}\right)$ such that

$$
X=\sum a_{I} T_{k}\left(e_{i}, 0, e_{j_{1}}, \cdots e_{j_{k}}\right) \in J .
$$


Now using (2) we conclude that

$$
T_{k}\left(e_{i}, 0, e_{j_{1}}, \cdots e_{j_{k}}\right) \in J \text { for some } I \text {. }
$$

For $m, n \neq i$ consider

$$
\left[T\left(e_{m}, e_{n}\right), T_{k}\left(e_{i}, 0, e_{j_{1}}, \cdots e_{j_{k}}\right)\right]=\ell \delta_{j_{i} m} T_{k}\left(e_{i}, e_{m}, e_{j_{1}}, \cdots \widehat{e}_{j_{i}}, \cdots e_{j_{k}}, e_{n}\right) \in J
$$

Now $T_{k}\left(e_{i}, e_{m}, e_{j_{1}}, \cdots \widehat{e}_{j_{i}}, \cdots e_{j_{k}}, e_{n}\right)=T_{k}\left(e_{i}, 0, e_{j_{1}}, \cdots \widehat{e}_{j_{i}}, \cdots e_{j_{k}}, e_{n}\right)$

$$
-T_{k+1}\left(e_{i}, 0, e_{j_{1}}, \cdots e_{j_{i}}, \cdots e_{j_{k}}, e_{n}\right)
$$

Now by (2) it follows that

$$
T_{k}\left(e_{i}, 0, e_{j_{1}}, \cdots \widehat{e}_{j_{i}}, \cdots e_{j_{k}}, e_{n}\right) \in J
$$

Now repeating this process we see that

$$
T_{k}\left(e_{i}, 0, e_{j_{1}}, \cdots e_{j_{k}}\right) \in J
$$

for all possible indices $j_{1}, \cdots j_{k}$ which are all different from $i$. Applying $T\left(e_{i}, e_{i}\right)$ to the above vector to conclude

$$
\begin{gathered}
T_{k}\left(e_{i}, e_{i}, e_{j_{1}}, \cdots e_{j_{k}}\right) \in J \\
T_{k}\left(e_{i}, 0, e_{j_{1}}, \cdots e_{j_{k}}\right)-T_{k}\left(e_{i}, e_{i}, e_{j_{1}}, \cdots e_{j_{k}}\right) \\
=-T_{k+1}\left(e_{i}, 0, e_{j_{1}}, \cdots e_{j_{k}}, e_{i}\right) \in J .
\end{gathered}
$$

Fix $j \neq i$. Replacing $k$ by $k+1$, consider the following vector which is in $J$ by $(*)$.

$$
\begin{gathered}
{\left[T\left(e_{j}, e_{i}\right), T_{k+1}\left(e_{i}, 0, e_{j_{1}}, \cdots, e_{j_{k+1}}\right)\right]=P \delta_{j j_{\ell}} T_{k+1}\left(e_{i}, e_{j}, e_{j_{1}}, \cdots \widehat{e}_{j_{\ell}}, \cdots e_{j_{k+1}}, e_{i}\right)} \\
-T_{k+1}\left(e_{j}, e_{i}, e_{j_{1}}, \cdots e_{j_{k+1}}\right) .
\end{gathered}
$$


Now $T_{k+1}\left(e_{i}, e_{j}, e_{j_{1}}, \cdots \widehat{e}_{j_{\ell}}, \cdots e_{j_{k+1}}, e_{i}\right)$

$$
=T_{k+1}\left(e_{i}, 0, e_{j_{1}}, \cdots \widehat{e}_{j_{\ell}}, \cdots e_{j_{k+1}}, e_{i}\right)-T_{k+2}\left(e_{i}, 0, e_{j_{1}}, \cdots e_{j}, \cdots e_{j_{k+1}}, e_{i}\right) .
$$

Now by $(* *)$ both vectors are in $J$. Thus we conclude that

$$
T_{k+1}\left(e_{j}, e_{i}, e_{j_{1}}, \cdots e_{j_{k+1}}\right) \in J .
$$

Now by (2) we see that

$$
T_{k+1}\left(e_{j}, 0, e_{j_{1}}, \cdots e_{j_{k+1}}\right) \in J .
$$

This is true for all possible indices $j_{1}, \cdots j_{k+1}$ which are all different from $i$.

Now applying $T\left(e_{j_{\ell}}, e_{i}\right)$ for $j_{\ell} \neq j$ we see that

$$
T_{k+1}\left(e_{j}, e_{j_{\ell}}, e_{j_{1}}, \cdots \widehat{e}_{j_{\ell}}, \cdots e_{j_{k+1}}, e_{i}\right) \in J
$$

Now by (2) we see that

$$
T_{k+1}\left(e_{j}, 0, e_{j_{1}}, \cdots, \widehat{e}_{j_{\ell}}, \cdots e_{j_{k+1}}, e_{i}\right) \in J
$$

Repeating this process we see that $T_{k+1}\left(e_{j}, 0, e_{\ell_{1}}, \cdots e_{\ell_{k+1}}\right) \in J$ for all possible $\ell_{1}, \cdots \ell_{k+1}$.

Now using the technique in the proof of Lemma 2.5 we see that

$$
T_{k+1}\left(e_{j}, 0, m_{1}, \cdots m_{k+1}\right) \in J \text { for all } m_{i} \in \Gamma
$$

Now replasing $k+1$ by $k+2$ we see that

$T_{k+2}\left(e_{j}, 0, m_{1}, \cdots m_{k+2}\right)=T_{k+1}\left(e_{j}, 0, m_{1}, \cdots m_{k+1}\right)-T_{k+1}\left(e_{j}, m_{k+2}, m_{1}, \cdots, m_{k+1}\right) \in J$.

Then it follows that

$$
T_{k+1}\left(e_{j}, m_{k+2}, m_{1}, \cdots m_{k+1}\right) \in J .
$$

Strictly speaking we have it for non-negative coefficients. But the other cases can be handled similarly. This proves $I_{k+1} \subseteq J$ and the Proposition. Further $s=0$. 
Proof of Theorem (1.9) . Let $V$ be a module as in Theorem. Let $V=\oplus_{r \in \Gamma} V_{r}$ be the weight space decomposition where

$$
V_{r}=\left\{v \in V \mid D(u, 0) v=(u, r+\alpha) v, \forall u \in \mathbb{C}^{d}\right\} .
$$

We know that $V_{r} \cong V_{s}$ as $T$-modules from Proposition 2.2(5). This with Proposition (3.3), Proposition (3.4) combined with the fact that some cofinite ideal of $T$ acts trivially on $V_{r}$ tells us that all $V_{r}^{\prime}$ s are isomorphic to some $V(\psi, b)$ as $g \ell_{d}$-modules. Note that the isomorphism between $V_{r}^{\prime} \mathrm{s}$ is given by $k(r)$ (from proof of proposition 2.2(5)). Thus if we let $V_{r}=V(\psi, b) \otimes t^{r}$ we see that $k(r) v(s)=v(s+r)$ for $v$ in $V(\psi, b)$.

Now consider $T(u, r)$ in $T / I_{2}$ and note that it is linear in both variables. Thus

$$
\begin{aligned}
T(u, r) v(s) & =\sum_{i, j} u_{i} r_{j} T\left(e_{i}, e_{j}\right) v(s) \\
& =\sum_{i, j} u_{i} r_{j} E_{j i} v(s)
\end{aligned}
$$

Therefore $k(-r) D(u, r) v(s)=D(u, 0) v(s)+\left(\sum u_{i} v_{j} E_{j i} v\right)(s)$

$$
=(u, s+\alpha) v(s)+\left(\sum u_{i} r_{j} E_{j i} v\right)(s) .
$$

Multiply both sides by $k(r)$ we get

$$
\begin{aligned}
D(u, r) v(s) & =(u, s+\alpha) v(s+r) \\
& +\left(\sum_{i j} u_{i} r_{j} E_{j i} v\right)(s+r) .
\end{aligned}
$$

This completes the proof of the theorem. 


\section{REFERENCES}

1. A.A. Belavin, A.M. Polyakov and A.B. Zamolodchikov, Infinite conformal symmetry in two dimensional quantum field theory, Nuclear Physics B 241, 333-380 (1984).

2. S. Berman, Y. Billig and J. Szmigiclski, Vertex operator algebras and the representation theory of toroidal Lie-algebras, Contemporary Mathematics, Vol. 297 1-26 (2002).

3. S. Berman and Y. Billig, Irreducible representations for toroidal Liealgebras, Journal of Algebra, 221, 188-231 (1999).

4. S. Eswara Rao, Irreducible representations of the Lie-algebra of the Diffeomorphisms of a $d$-dimensional torus, Journal of Algebra, 182, 401-421 (1996).

5. S. Eswara Rao, Generalized Virasoro operators (2004). To appear in Communications in Algebra.

6. S. Eswara Rao and R.V. Moody, Vertex representations for n-toroidal Lie algebras and a generalization of the Virasoro algebra, Comm. Math. Physics 159, 239-264 (1994).

7. S. Eswara Rao and C. Jiang, Classification of irreducible integrable representations for the full toroidal Lie-algebras, preprint 2004.

8. P. Goddard and D. Olive, Kac-Moody and Virasoro algebras, World Scientific, Singapore (1988).

9. F. Figueirido and E. Ramos, Fock space representation of the algebra of diffeomosphisms of the n-torus, Physics letters B, 238, 247-251 (1990). 
10. P. Di Francesco, P. Mathieu and D. Senechal, Conformal Field Theory, Springer Verlag, New York (1997).

11. C Jiang and D. Meng, Integrable representations for generalised Virasorotoroidal Lie-algebra, 270, 307-334 (2003).

12. V. Kac and I. Frenkel, Basic representation of affine Lie algebras and dual resonanace models, Invent. Math. 62, 23-66 (1980).

13. V. Kac and A.K. Raina, Bombay lectures on highest weight representations of infinite dimensional Lie-algebras, World Scientific, Singapore, (1987).

14. T.A. Larsson, Multi dimensional Virasoro algebra, Physics Letters B, 231, 94-96 (1989).

15. T.A. Larsson, Central and non-central extensions of multi-graded Lie algebras, Journal of Physics A, 25, 1177-1184 (1992).

16. T.A. Larsson, Conformal fields: A Class of Representations of Vect $(N)$, Internat. J. Modern Phys. A. Vol 7, No.26, 6493-6508 (1992).

17. T.A. Larsson, Lowest energy representations of non-centrally extended diffeomorphism algebras, Comm. Math. Physics, 201, 461-470 (1999).

18. T.A. Larsson, Extended diffeomorphism algebras and trajectories in jet space, Comm. Math. Physics, 214, 469-491 (2000).

19. O. Mathieu, Classification of Harish-Chandra Modules over the Virasoro Algebras, Invent. Math. 107, 225-234 (1992).

20. R.V. Moody, S. Eswara Rao and T. Yokomuma, Toroidal Lie algebras and vertex representations, Geom. Ded. 35, 283-307 (1990). 
21. E. Ramos, C.H. Sah and R.E. Shrock, Algebra of diffeomorphisms of the $N$-torus, J. Math. Phys.31, No.8., 1805-1816 (1990).

22. E. Ragoucy and P. Sorba, An attempt to relate area-preserving diffeomorphisms to Kac-Moody Lie algebras, Letters in Mathematical Physics, 21, 329-342 (1991).

23. E. Ramos and R.E. Shrock, Infinite dimensional $\mathbb{Z}^{n}$ - indexed Lie algebras and their super symmetric generalizations, International Jour. of Modern Phys. A, Vol.4, No. 16, 4295-4302 (1989).

24. Yuly Billig, Energy-momentum tensor for the toroidal Lie algebras, arXiv. Math. RT/0201313 (2002). 